\title{
A Hybrid Genetic Algorithm and Vector Fitting Based Approach for Approximation of Propagation Function of Transmission Lines
}

\author{
Yue $\mathrm{Yu}^{1}$, Qingyu $\mathrm{Ge}^{2 *}$ and Chongqing Jiao ${ }^{2}$ \\ ${ }^{1}$ State Key Laboratory of Power Grid Safety and Energy Conservation, Relay Protection Research Department, China Electric Power \\ Research Institute, Beijing, 100192, China \\ ${ }^{2}$ State Key Laboratory of Alternate Electric Power System with Renewable Energy Sources, North China Electric Power University, \\ Beijing, 102206, China
}

\begin{abstract}
A hybrid genetic algorithm and vector fitting based approach is proposed for the approximation of the propagation function of transmission lines. In this work, the process of guessing the number of poles and then adjusting corresponding delay times in the conventional method is improved by the genetic algorithm. The proposed approach can result in a better approximation of propagation function, which is proven by a successful implementation to an overhead single core coaxial cable.
\end{abstract}

\section{Introduction}

A transmission line can be characterized by two matrix transfer functions: the propagation function $\boldsymbol{H}$ and the characteristic admittance $\boldsymbol{Y}_{\mathrm{C}}$. These two quantities are frequency-dependent and can only be calculated as discrete functions in the frequency domain. A timedomain simulation can be carried out using convolutions, which involves the time-domain counterparts of $\boldsymbol{H}$ and $\boldsymbol{Y}_{\mathrm{C}}$ obtained via an inverse Fourier transform. One of the major problems with time-domain analysis is the inability to acquire accurate frequency-domain descriptions of $\boldsymbol{H}$ and $\boldsymbol{Y}_{\mathrm{C}}$ with rational functions. Nowadays, the Universal Line Model (ULM) is a widely used phase model, which utilizes Vector Fitting (VF) to identify the system in the frequency domain [1-2]. VF is a robust reformulation of the Sanathanan-Koerner iteration [3]. It utilizes pole relocation instead of weighting and partial fractions as rational basis functions instead of polynomials [4].

However, it is difficult to fit $\boldsymbol{H}$ because the elements of $\boldsymbol{H}$ are subject to different modes and time delays. Failure to identify the delay time of the system accurately may deteriorate the accuracy of the approximation. Particularly, it affects the necessary number of poles to approximate the functions. Besides, the number of poles cannot be decided directly. Conventionally, the number of poles is increased associated with a corresponding delay time until reaching a satisfying result, which cannot always guarantee the best result. In this paper, a genetic algorithm (GA) proposed in [5] is implemented for the optimization of the number of poles and the delay time.

\section{Problem formulation}

In ULM, the voltage and current relationship at the ends of a transmission line is given by

$$
\begin{aligned}
& \dot{I}_{\mathrm{L}}-\boldsymbol{Y}_{\mathrm{C}} \dot{\boldsymbol{U}}_{\mathrm{L}}=-\boldsymbol{H}\left[\dot{\boldsymbol{I}}_{0}+\boldsymbol{Y}_{\mathrm{C}} \dot{\boldsymbol{U}}_{0}\right] \\
& \dot{\boldsymbol{I}}_{0}-\boldsymbol{Y}_{\mathrm{C}} \dot{\boldsymbol{U}}_{0}=-\boldsymbol{H}\left[\dot{\boldsymbol{I}}_{\mathrm{L}}+\boldsymbol{Y}_{\mathrm{C}} \dot{\boldsymbol{U}}_{\mathrm{L}}\right]
\end{aligned}
$$

where all quantities are in the frequency domain. The matrices for propagation $\boldsymbol{H}$ can be expressed as

$$
\boldsymbol{H}=e^{-\sqrt{\boldsymbol{Y Z}} L}
$$

$\boldsymbol{Z}$ and $\boldsymbol{Y}$ are the series impedance and shunt admittance per unit length, and $L$ is the length of the transmission line. For an $n$ conductor system, $\boldsymbol{Z}$ and $\boldsymbol{Y}$ are $n \times n$ matrices.

\subsection{Rational function approximation of propagation function}

The propagation function of a transmission line, such as overhead lines or cables, may be expressed as a rational polynomial in terms of its poles and zeros, that to be computationally tractable, we need to approximate by only the dominant poles and zeros of the function. The final approximation must provide the time-frequency behavior of the original function. The major difficulty here is that its elements could involve a mix of up to $N$ different delay terms due to the multimode propagation on an $N$-conductor line. Usually, modal delays can be grouped according to almost identical values. Suppose

*Corresponding author's e-mail: 1182201150@ncepu.edu.cn 
there are $N_{g}$ groups of delay times and propagation matrix $\boldsymbol{H}$ can be made up of all modes:

$$
\boldsymbol{H}=\sum_{k=1}^{N_{g}} \widetilde{\boldsymbol{H}}_{k} e^{-s \tau_{k}}
$$

where $N_{g} \leq N$, and $\tau_{k}$ is the delay for the $k$-th group. Each term $\widetilde{\boldsymbol{H}}_{k}$ at (4) can now be considered free of delay factors and can be fitted as follows:

$$
\widetilde{\boldsymbol{H}}_{k}=\sum_{i=1}^{N_{h}(k)} \frac{\widetilde{\boldsymbol{R}}_{k, i}}{s-p_{k, i}} k=1,2, \ldots, N_{g}
$$

where $N_{h}(k)$ is the fitting order for the $k$-th term $\widetilde{\boldsymbol{H}}_{k}, p_{k, i}$ represents its $i$-th fitting pole, and $\widetilde{\boldsymbol{R}}_{k, i}$ is the corresponding matrix of residues. With poles and time delays of all modes, the following rational form is obtained for $\boldsymbol{H}$ :

$$
\boldsymbol{H}=\sum_{k=1}^{N_{g}} e^{-s \tau_{k}} \sum_{i=1}^{N_{h}(k)} \frac{\boldsymbol{R}_{k, i}}{s-p_{k, i}}
$$

where $\widetilde{\boldsymbol{R}}_{k, i}$ is the matrix of residues for $\boldsymbol{H}$.

\subsection{Conventional identification algorithm}

Two fitting error tolerances are defined first for the approximation of $\boldsymbol{H}$ and $\widetilde{\boldsymbol{H}}_{k}$, respectively. Then, the time delays and poles are estimated in the modal domain. The number of poles needs to be increased from 1 to maximum and the poles result from applying VF to the modal propagation conforming each delay group. The search runs until the modal fitting error is under desired tolerance. With all the poles $p_{k, i}$ and group delays $\tau_{k}$ of (6) being obtained in the modal domain, the unknown residues of $\boldsymbol{H}$ are calculated by solving an overdetermined linear equation as a least-squares problem. A necessary check is called up here to make sure that fitting error is under the desired tolerance and the tolerance for $\widetilde{\boldsymbol{H}}_{k}$ will be reduced by half to enhance the accuracy of modal contribution. The process will be repeated until the fitting error in the phase domain is accepted. Sometimes, model order reduction is needed if a large ratio of residue over pole exists which may disturb simulation in the time domain, which means certain poles will be removed to assure steady simulation. Thus, the overall fitting of $\boldsymbol{H}$ is completed.

\section{Optimization using genetic algorithms}

GA is applied to fitting propagation function $\boldsymbol{H}$ to substitute the process of increasing the number of poles to get satisfying modal poles and delay times. The parameters to be optimized in the GA are the number of poles $n$ and modal delay time $\tau$. Particularly, $n$ is an integer, which is bounded between 1 and maximum; and $\tau$ is a continuous variable, which is bounded between maximum value $L / c$ ( $L$ is the length of transmission line and $c$ is the velocity of light) and minimum value $L / v_{\omega}$ ( $v_{\omega}$ represents the velocity of the high-frequency component). To implement GA, some basic definitions need to be clarified in the following.

\subsection{Initial population}

An initial population made up of individuals is created randomly at the beginning and then evolves. Each individual, a string of binary bits, represents a $(n, \tau)$ pair that comes from encoding a candidate solution vector $[n, \tau]$. The choice of population size is always a trade-off between efficiency and effectiveness [6]. For our application, we choose a population size of 20 individuals.

\subsection{Crossover}

An individual is selected and crossed with another one. Several bits or genes at the same coordinate from these two individuals are exchanged, resulting in offspring individuals. The same procedure is followed for all individuals to make a new double-sized population.

\subsection{Mutation}

Mutation introduces additional random changes to genes of selected individuals with a predefined probability. This maintains diversity within the population during evolution. In our work, a dynamic approach for the probability of mutation is adopted for a better exploration of the solution. Particularly, a comparatively large value and range for the initial generations whereas a smaller value and range at following generations for fast convergence.

\subsection{Fitness Evaluation}

The binary population is decoded and normalized by applying boundary conditions. In every run, the GA calls the VF to approximate modal propagation $\boldsymbol{H}_{k}$ using those $(n, \tau)$ as input and calculate fitting errors. A comparison of recorded fitting errors with the desired error tolerance gives the deviation $\delta$ as follows:

$$
\delta=|e-t o l|
$$

where $e$ indicates the max fitting error of approximation of modal propagation, and tol indicates the specified tolerance. The best fitness value for a population is the smallest value for any individual in the population because the minimum deviation is expected.

\subsection{Selection}

The individuals are sorted from smallest to largest by its fitness value. The best half is selected for the next generation. Since the crossover operator doubled the original population, this operator resizes the population to its original size. 


\subsection{Termination Condition}

Fitness values of the best individuals in five consecutive generations are recorded in the procedure. The algorithm is terminated if the average relative change in the fitness function value over adjacent generations is less than defined tolerance.

\section{Simulation result}

The proposed method was implemented on an overhead single core coaxial cable, which consists of an inner conductor, a shield, and an outer sheath. It's more difficult to fit the propagation function for cables than overhead lines because cables are more seriously frequency- dependent. The initial tolerance for the approximation of the modal propagation function is 0.25 and the tolerance for phase propagation function is 0.02 . After tolerance for modal fitting being reduced 6 times and model order reduction due to large residue-pole ratio, the fitting of the propagation function was done in the phase domain. A comparison with the conventional method is shown in Figure 1, Figure 2, and Table 1. As shown in Figure 1 and Figure 2, we got more fitted points placed on the original curves by the proposed approach, especially in high frequency. It's because more poles are reserved after model order reduction. From Table 1, the result from the proposed method is far more accurate than that from the conventional method, and correspondingly, the delay times from the proposed method are more reliable.

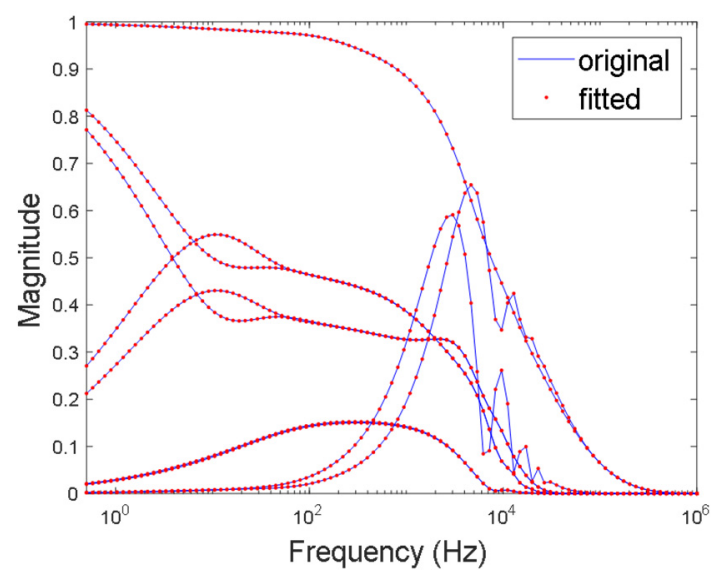

(a)

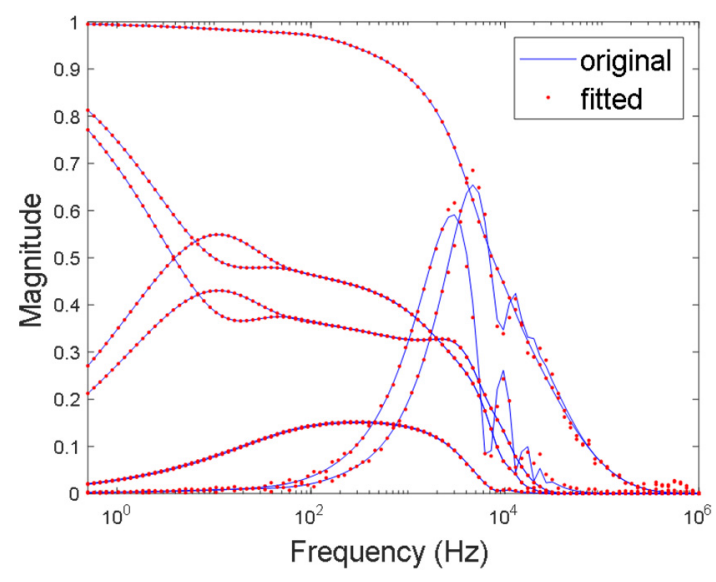

(b)

Figure 1. Magnitudes of elements in $\boldsymbol{H}$ : (a) proposed method with GA; (b) conventional method.

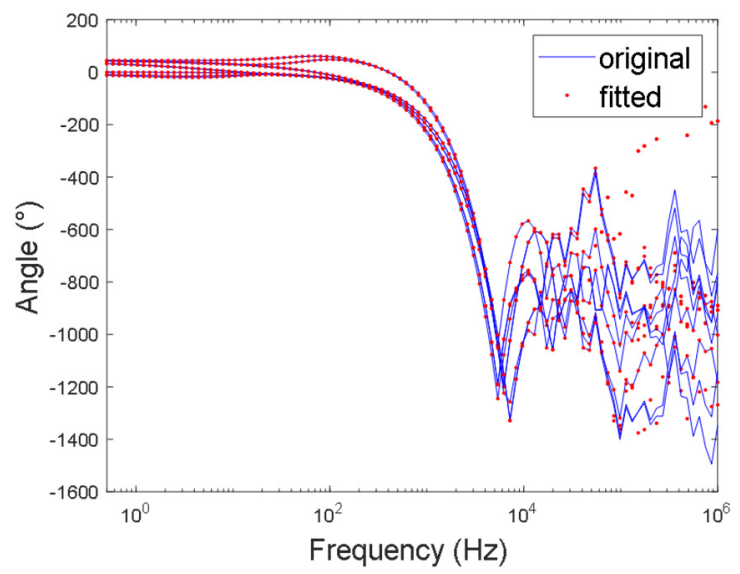

(a)

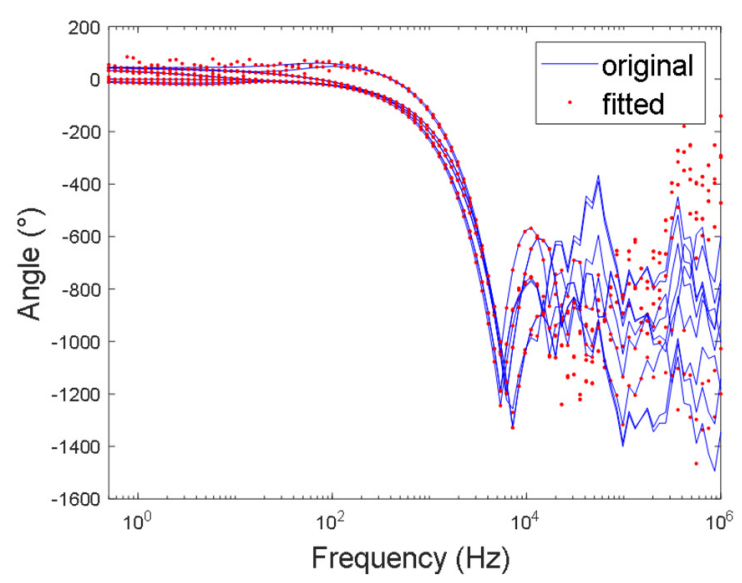

(b)

Figure 2. Angles of elements in $\boldsymbol{H}$ : (a) proposed method with GA; (b) conventional method.

Table 1. Comparison of the proposed method and the conventional method.

\begin{tabular}{|c|c|c|c|c|c|}
\hline & \multirow{2}{*}{$\begin{array}{l}\text { Fitting } \\
\text { error }\end{array}$} & \multirow{2}{*}{ Number of poles } & \multicolumn{3}{|c|}{ Delay time (ms) } \\
\hline & & & $\tau_{1}$ & $\tau_{2}$ & $\tau_{3}$ \\
\hline Proposed Method & $4.05 \mathrm{e}-4$ & 132 & 0.6468 & 0.6243 & 0.4663 \\
\hline Conventional Method & $1.50 \mathrm{e}-2$ & 119 & 1.1994 & 0.5133 & 0.4264 \\
\hline
\end{tabular}




\section{Conclusion}

This paper has proposed a hybrid GA and VF based approach for approximation of the propagation function of transmission lines. It's proven that the performance of approximation can be improved when GA is introduced to the conventional method. Successful implementation of such a method to an overhead single core cable shows the feasibility and accuracy of the proposed approach.

\section{Acknowledgments}

This project was supported by the Science and Technology Project of SGCC "Research on Outdoor Installation Protection Scheme and Key Technologies."

\section{References}

1. Morched, A., Gustavsen, B., Tartibi, M. (1999) A Universal Model for Accurate Calculation of Electromagnetic Transients on Overhead Lines and Underground Cables. IEEE Transactions on Power Delivery, 14(3): 1032-1038.

2. Gustavsen, B., Irwin, G., Mangelrød, R., et al. (1999). Transmission Line Models for the Simulation of Interaction Phenomena Between Parallel Ac and Dc Overhead Lines. In: International Conference on Power System Transients. Budapest. pp. 61-67.

3. Sanathanan, C., Koerner, J. (1963) Transfer Function Synthesis as a Ratio of Two Complex Polynomials. IEEE Transactions on Automatic Control, 8(1): 56-58.

4. Hendrickx, W., Dhaene, T. (2006) A Discussion of "rational Approximation of Frequency Domain Responses by Vector Fitting". IEEE Transactions on Power Systems, 21(1): 441-443.

5. Holland, J. (1975) Adaptation in Natural and Artificial Systems: An Introductory Analysis with Applications to Biology. University of Michigan Press.

6. Reeves, C. R. (2010) Genetic algorithms. In: Gendreau, M. and Potvin, J. Y. (Eds), Handbook of Metaheuristics. Springer, New York. pp. 109-139. 